\title{
Impact of experiencing specific side-effects on contraceptive switching and discontinuation in Uganda: Results from a longitudinal PMA Survey
}

Linnea A Zimmerman ( $\square$ linnea.zimmerman@jhu.edu ) Johns Hopkins University Bloomberg School of Public Health https://orcid.org/0000-0002-0118-0889

Dana Sarnak

Johns Hopkins University Bloomberg School of Public Health

Celia Karp

Johns Hopkins University Bloomberg School of Public Health

Shannon Wood

Johns Hopkins University Bloomberg School of Public Health

Saifuddin Ahmed

Johns Hopkins University Bloomberg School of Public Health https://orcid.org/0000-0002-9884-4580

Fredrick Makumbi

Makerere University https://orcid.org/0000-0002-6460-7325

Simon Kibira

Makerere University https://orcid.org/0000-0002-7385-423X

\section{Research}

Keywords: Contraceptive side-effects, contraceptive continuation, contraceptive switching, longitudinal, menstrual bleeding, sexual experience, Uganda

Posted Date: November 6th, 2020

DOI: https://doi.org/10.21203/rs.3.rs-47447/v2

License: (a) This work is licensed under a Creative Commons Attribution 4.0 International License. Read Full License 


\section{Abstract}

There is substantial evidence that contraceptive side-effects are a major deterrent to consistent use of contraception but few studies in low- or middle-income countries explore the role that specific sideeffects, rather than "side-effects and health concerns", have on contraceptive dynamics. This study used population-based, longitudinal data to explore the effect that specific side-effects had on contraceptive continuation, discontinuation, and switching in Uganda.

Methods Data for this study come from two rounds of PMA2020 data collection in Uganda PMA2020 Uganda's sixth cross-sectional survey and a follow-up survey conducted one year later. The main outcomes of interest were discontinuation and switching among modern users of hormonal contraceptive methods and the IUD at baseline $(n=550)$. Multivariable multinomial logistic regressions assessed the association of reporting experiencing specific side-effects (more bleeding, less bleeding, irregular bleeding, increased dryness/reduced libido, and physical discomfort) with discontinuation and switching one year later, adjusting for socio-demographic characteristics, type of method, and length of use.

\section{Results}

One-third of hormonal contraceptive users reported experiencing side-effects at baseline. Reporting more bleeding increased the risk of discontinuation by 2.39 times (95\% Cl: 0.01-4.72). Experiencing irregular bleeding decreased the risk of switching by a factor of 0.40 (95\% Cl: 0.17-0.93). Vaginal dryness/reduced libido and physical discomfort were both marginally associated with increased relative risk of switching, increasing the risk of switching by a factor of 3.10 (95\% Cl : 0.97-9.10) and 1.75 (95\% Cl: 0.95-3.24), respectively. Wealth was significantly negatively associated with discontinuation while education was marginally significantly and positively associated with switching.

\section{Conclusions}

Greater attention should be paid to understanding the unique contributions of side-effects to contraceptive behavior using population-based data. Not all side-effects are associated with contraceptive discontinuation and switching, despite the experience of side-effects being common. The experience of physical discomfort and vaginal dryness/reduced libido, largely ignored in other research, were shown to affect use. Providing greater individualized care that includes information about common side-effects and how they may impact daily life is necessary.

\section{Plain English Summary}

Research has shown that experiencing side-effects is related to stopping use of contraception, even when women wish to avoid pregnancy. Most research, however, does not differentiate between different sideeffects, such as increased bleeding or changes to sexual pleasure, instead combining all into "side-effects or health concerns". We used data from 550 women in Uganda, who were interviewed twice, one year 
apart, to see if women who reported different side-effects at the first interview were more likely to stop using contraception or switch to a different contraceptive method than women who did not report experiencing side-effects. We found that increased menstrual bleeding was associated with a higher rate of contraceptive discontinuation, while women who reported vaginal dryness/reduced libido or who reported physical discomfort, such as cramping, were more likely to switch methods than women who did not report these issues. It is important to understand what side-effects are likely to motivate stopping versus switching contraception so that education and counseling can inform women of side-effects they may experience, help them choose the best method based on what side-effects they deem important, and if necessary, aid in switching contraceptive methods.

\section{Background}

There is substantial evidence that contraceptive side-effects, either experienced or perceived, are a major deterrent to consistent use of contraception. The majority of women who discontinue contraception while still wanting to delay or avoid pregnancy do so for "method-related concerns" (1,2); Bradley and colleagues found that among women who discontinued while still in need of a method, as many as $37 \%$ reported side-effects as their primary reason for discontinuation. Prospective studies and clinical trials have documented a number of side-effects arising from hormonal contraceptives and the non-hormonal copper IUD: disruptions to menstrual bleeding, breast tenderness, nausea, acne, headaches, dizziness, and changes in sexual desire (3-10). Though the wide range of side-effects is well established, there has only recently been an effort to understand how each of these side-effects are related to overall contraceptive use dynamics - adoption, discontinuation, and switching.

The majority of research that has highlighted the importance of specific side-effects in LMICs has been qualitative. Across diverse settings, women report experiencing changes to menstrual bleeding, abdominal pain, weight gain, changes to sexual experience, and non-specific "weakness" as influencing their decision-making about contraception (11-18). Though providing critical contextual data, qualitative data are unable to assess how widespread experiences are, how these experiences contribute to contraceptive use dynamics at a population level, and what side-effects may be largely tolerated by women versus those that lead to discontinuation or switching.

None of the population-based national surveys that constitute the majority of publicly available data on family planning, such as the DHS, PMA2020, and MICS, include questions that identify specific sideeffects, instead identifying only generalized "side-effects" or "health concerns" as a reason for contraceptive discontinuation $(14,19,20)$; however, recent efforts have attempted to expand our understanding of specific side-effects. While changes to menstrual bleeding, including increased bleeding, amenorrhea, and irregular bleeding and spotting, have been shown to be particularly influential on contraceptive use $(1,8,21)$, a recent review highlighted the lack of nationally representative data 
available on the experience of these side-effects (21). A recent review of contraceptive-induced changes to sexual experience similarly noted both the importance that is placed on these changes by women and the critical dearth of research in this area in low- and middle-income countries (LMICs) (22). Similar gaps in knowledge exist around the prevalence and consequences of other specific side-effects that have been raised in qualitative research, such as changes to sexual experience, naudea, and headaches. This lack of knowledge limits our understanding of both the prevalence and effect of specific contraceptive induced side-effects on contraceptive use behavior.

Beyond understanding what the prevalence of side-effects may be in a population, it is also important to understand what factors may influence the tolerability of side-effects; that is, what factors are associated with women continuing to use a method in the presence of side-effects. This question is particularly relevant to consider in the context of LMICs, as the vast majority of research has been limited to highincome settings or conducted as part of clinical trials $(3,6,7,23-25)$. Tolerability of specific side-effects may differ for a variety of social, cultural, and economic reasons; how side-effects are interpreted, treated (e.g. availability and accessibility to menstrual hygiene products or treatment for cramping), and the economic and physical consequences when they are experienced are heavily influenced by context and quality of available services $(12,26,27)$. Findings from high-income countries should not, therefore, be applied to LMIC contexts nor should findings from one LMIC be rotely applied to another. As Abdel-Tawab and RamaRao discuss, improvements to contraceptive services, which aim to address these embodied experiences, must be informed by context and modified to be contextually appropriate (27). Gaining a more nuanced understanding of the characteristics of women in specific settings who tolerate sideeffects and continue using contraception, versus those that discontinue, may thus provide valuable lessons to program managers and health providers.

Finally, limited research has explored how the experience of specific side-effects may motivate switching to a different method versus discontinuing contraception altogether. Contraceptive switching has significant programmatic implications for 1) promoting the use of more-effective, long-acting methods from lesser effective methods; 2) addressing discontinuation related to experienced side-effects by promoting alternative contraceptive methods; 3 ) ensuring that women are able to choose a method that best aligns with their reproductive goals-a critical component of high-quality, rights-based family planning programs (28-31). While the outcome of switching is generally considered positive in terms of sustained protection against unintended pregnancy, there is significantly less known about what motivates switching versus discontinuation and what facilitates or impedes this decision. This lack of knowledge limits the ability of family planning programs to address women's concerns that motivate switching to less effective methods. Increased research in this area will aid in improving programmatic approaches to help women achieve their reproductive goals. To our knowledge, the only prospective study in LMICs to assess specific side-effects and their effect on contraceptive switching versus 
discontinuation was conducted by Barden-O'Fallon and colleagues among women in Honduras (32). The authors found that Honduran women who experienced amenorrhea or heavy menstrual bleeding were significantly more likely to discontinue than switch methods and that urban women were significantly more likely to switch methods than rural women, underscoring the simultaneous roles of side-effects and sociodemographic characteristics on women's contraceptive practices. However, the study did not compare switching and discontinuing separately to assess the effect of experiencing side-effects on these behaviors, relative to continuation. Despite the logical contribution of side-effects to women's decisions to adopt a different method or stop using contraception entirely, there is a clear dearth of research in this area.

To better understand the prevalence of different side-effects and their association with contraceptive use dynamics, a longitudinal study was conducted in Uganda. This study aimed to estimate the prevalence of a range of specific side-effects that were experienced among users of hormonal contraception and to establish the association between these side-effects and contraceptive discontinuation, switching, or continuation one year later. Additionally, we sought to identify relevant socio-demographic characteristics associated with discontinuation and with switching.

\section{Methods}

\section{Study Setting}

Uganda had a population of about 44.3 million in 2019, with nearly half its population under age 15 (33). Uganda is characterized by high fertility; the total fertility rate (TFR) in 2016 was 5.4 births, down from 6.9 in 2000-2001 (19). Modern contraceptive use in Uganda has also increased in recent years, from $21 \%$ in 2014 to $30 \%$ in 2018. Injectables were the most common method used in 2018 (39\%), followed by implants (20\%) (ibid). Despite the increase in contraceptive use, high rates of contraceptive discontinuation persist; nationally, $20 \%$ of IUD users, $17 \%$ of implant users, $36 \%$ of injectable users, and $46 \%$ of pill users discontinued in the first year of use, while in need of protection against unintended pregnancy.

\section{Study Overview and Sampling}

Data for this study come from two rounds of Performance Monitoring and Accountability (PMA2020) data collection in Uganda. Specifically, in PMA2020 Uganda's sixth cross-sectional survey (20), conducted from April to June 2018 (hereafter referred to as baseline), the consent and design were modified to enroll women in a longitudinal study. A follow-up survey was conducted approximately after one-year from May to June 2019. 
The PMA2020 is a multi-stage cluster, nationally representative survey of women age $15-49$. In the baseline survey, 110 Enumeration Areas (EA) were selected using probability proportional to size sampling and all occupied households were enumerated. Forty-four households were randomly selected within each EA, consented, and interviewed. All women age 15-49 who were either usual members of the household or who slept in the household the night before were approached for interview, and if consented, interviewed by a trained interviewer. A total sample of 4,288 women were interviewed in the baseline survey (response rate: $96.9 \%)$; the majority $(95.5 \% ; n=4,095)$ of women agreed to participate in the follow-up survey. Further information on the design of PMA2020 surveys is available from www.pmadata.org and Zimmerman et al (2017).

At follow-up, interviewers returned to the households of women who completed the baseline survey and re-consented women to participate in the follow-up survey. We were able to relocate and successfully interview 2,755 of the original sample, resulting in a follow-up rate of $67 \%$. Due to potential bias from loss to follow-up, we constructed an inverse probability weight from estimated propensity scores to adjust differential loss to follow-up. Using the total sample of women from baseline $(n=4,288)$, we adjusted for the probability of being interviewed at follow-up accounting for age (five-year age groups), education (none, primary, secondary and higher) , marital status (currently married/in-union, not married), wealth (five quintiles), and residence (urban,rural). The original baseline individual female weight was then multipled by the inverse probability weight to construct the final weight in the analyses. No significant differences in baseline characteristics between all women in the baseline and those who were successfully followed-up were detected after the application of the final sampling weights.

\section{Analytic Sample}

This analysis was restricted to women who reported currently using hormonal contraception (implant, injectable, pill) and IUD at baseline. Of note, we are unable to differentiate between hormonal and nonhormonal IUD use. Users of emergency contraception were excluded due to the periodicity in its use and female sterilization users were excluded as they would not be able to discontinue or switch methods. Condoms and other barrier and traditional methods were excluded as systemic side-effects were not anticipated from using these methods. In total, 550 women were included in this analysis; the flowchart of sample selection process is shown in Figure 1. Data was complete for all observations used in analysis. The follow-up rate for the analytic sample was also 67\%; 550 users were relocated out of 819 pill, implant, injectable, and IUD users at baseline.

\section{Measures}


At baseline, women were asked to report whether they were currently experiencing any side-effects, and if so, to specify all that they were currently experiencing (with multiple-response options; responses were not read aloud). The list of side-effects was drawn from the literature on clinically documented sideeffects, and those that have been reported in qualitative research in Uganda. The list was reviewed with the in-country team and pilot tested; the complete list of side-effects and frequencies with which they were reported is shown in Appendix 1.

The outcomes related to contraceptive behavior were defined based on the woman's contraceptive use status at the time of follow-up survey. Specifically, women were categorized in to three groups: either continuing (using the same method at baseline and follow-up), switched (using a different method at baseline and follow-up), or discontinued (not using a method at follow-up). We assessed the distribution of each outcome by method type (implant, IUD, injectables, and oral pills).

\section{Covariates}

All analyses adjusted for the following socio-demographic variables measured at baseline: age (categorical variable of 15-24, 25-34, and 35 and above), marital status (binary variable indicating married/in-union), residence (urban or rural), education (categorical variable indicating none, primary, or secondary and above), and parity (categorical variable of $0-1,2-3$, or $4+$ children). We combined nulliparous and primiparous women, as there were only nine nulliparous women included in the analytic sample. Due to sample size limitations, we created a binary variable for wealth, indicating whether the respondent resided in a household in one of the lowest two wealth quintiles (0) or the wealthier three (1). To account for the fact that side-effects may be more frequent when initiating a method and resolve over time, we included a binary variable indicating whether the woman started her method less than 12 months prior to the baseline interview. We used a 12-month time frame to align with previous research (32). We adjusted for method used at baseline with a binary variable indicating short-acting (pill and injectable) or long-acting method (IUD and implant). As the mechanism of discontinuation differs between short- and long-acting methods (i.e. a clinical visit is necessary to stop using either the implant, the hormonal or the non-hormonal IUD, but not necessary for the pill or injectable), we felt that it was important to understand whether the risk of discontinuation and switching is modified by the method type. Finally, we adjusted for stated fertility intentions at baseline (wanted child within two years, wanted child after two years, wanted no more children). The purpose of our analysis is to assess the effect of experiencing side-effects at the population level of contraceptive users, thus, we adjusted for, but did not exclude observations, based on women's reported fertility intentions. It is possible that women are more likely to tolerate side-effects when their desire to control their fertility is more pronounced, for example, women who want no more children, and thus, we felt the inclusion of fertility intentions in the model was critical. Using the full sample also enables us to make inferences about the results at the population level rather than for a sub-group of women who wish to avoid or delay a pregnancy. 
Exploratory analyses assessed the prevalence of each side-effect for further modeling considerations. Based on sample size, the following side-effects were combined: "uterine cramping", "cramping", "headache", "nausea", and "weakness" ("physical discomfort"); "vaginal dryness" and "lowered libido" ("vaginal dryness/lowered libido"); "spotting" and "irregular bleeding" ("irregular bleeding").

Only side-effects that were reported by a minimum of 20 women were included in models. Unadjusted multinomial models assessed the risk of discontinuing or switching, relative to continuing, among women who reported experiencing the side-effect compared to women who did not. As women could experience multiple side-effects at once, we ran adjusted multinomial models that included all sideeffects and the covariates listed above. Sensitivity analyses were conducted with the inclusion of only one side-effect at a time and results were largely consistent (Appendix 2 and 3 ).

Descriptive analyses (Table 1- Table 3 below ) applied the survey weights described above. Due to small sample sizes and because weighting increases variances and design-effects substantially, we tested the efficiency of the application of weights in the multinomial regressions. The inefficiency index (34) of each unadjusted multinomial model assessing the relationship of each side-effect ranged from $8 \%$ to $56 \%$ with a median of $30 \%$. To maximize efficiency, we followed guidance from Korn and Graubard and did not apply the survey weights to the regression multinomial models (34); we did, however, adjust for clustering using design-based analysis. All analyses were conducted using Stata v16.0 (35). We used the STROBE cohort checklist when writing our report (36).

\section{Result}

The characteristics of women in the analytic sample are shown in Table 1. The average age of women in the sample was 29.3 and the majority of women (81.3\%) were married or in-union. One out of five users was nulliparous or primiparous and the vast majority (94.1\%) had attended at least some schooling. About $24 \%$ of current users reported wanting a child within two years. About half of women reported that they had been using the current hormonal contraceptive method for fewer than 12 months. The majority of women were using the injectable (52.0\%) followed by the implant (34.0\%).

Table 2 shows the percentage of women who continued the same method at follow-up interview, switched, or discontinued the method that they were using at baseline by method type; in total, $38.0 \%$ of users were using the same method at follow-up; $25.2 \%$ had switched methods; and $36.8 \%$ had 
discontinued use. Switching was highest among pill users, while discontinuation was highest among injectable users.

Table 3 shows the percentage of women who reported each side-effect at baseline among all women and by continuation status at follow-up. Slightly more than one-third of the sample reported experiencing any side-effect at baseline (35.3\%). Among all women, physical discomfort was the most commonly reported side-effect (15.4\%). Among women who continued, $29.9 \%$ reported experiencing any side effect at bseline, while $39.6 \%$ of women who discontinued and $37.2 \%$ of women who switched reported any side-effect at baseline. Among women who continued and among women who switched, physical discomfort was reported most frequently (12.8\% and $18.5 \%$, respectively), while among women who discontinued, less bleeding was most frequently reported (17.1\%).

The crude and adjusted relative risk ratios for discontinuation and switching relative to continuation are shown in Table 4 below. As the adjusted model included all side-effects, we do not show a separate adjusted model for reporting any side-effect; the crude model, however, shows an increased risk of discontinuation, though not switching, among women who reported any side-effect (cRRR: 1.55, 95\% Cl: 1.10-2.20). Reporting more bleeding increased the risk of discontinuation by 2.39 times ( $95 \% \mathrm{Cl}$ : 1.21 4.72). Though irregular bleeding was not statistically significantly associated with switching in the crude models, after adjustment, experiencing irregular bleeding decreased the risk of switching by a factor of 0.40 (95\% Cl: 0.17-0.93). Reporting vaginal dryness/lowered libido and physical discomfort were both marginally associated with increased relative risk of switching; reporting vaginal dryness and/or lowered libido increased the relative risk of switching by a factor of $3.10(95 \% \mathrm{Cl}: 0.97-9.90)$ and physical discomfort increased the relative risk by a factor of 1.75 (95\% Cl: $0.95-3.24)$.

The risks of discontinuing and switching were significantly higher among women using short-acting methods (aRRR: $3.90 ; 95 \%$ Cl: 2.44-6.24 and aRRR: $6.31 ; 95 \% \mathrm{Cl}$ : 3.52-11.31, respectively) relative to women using a long-acting method. Wealth and fertility desires were significantly associated with discontinuation, with wealthier women significantly less likely to discontinue (aRRR: 0.57 ; $95 \%$ Cl: $0.34-$ 0.96). Relative to women who reported wanting a child within the next two years, women who did not want a child in the next two years (aRRR: $0.33 ; 95 \% \mathrm{Cl}$ : 0.16-0.67) or who wanted no more children (aRRR: $0.21 ; 95 \% \mathrm{Cl}: 0.09-0.45)$ were significantly less likely to discontinue use one year later. Age and education were marginally associated with increased relative risk of switching, though having either primary or secondary education appeared to increase the risk of switching by approximately a factor of 3 (aRRR: 3.06; 95\% Cl: 0.89-10.50 and aRRR: 3.47, 95\% Cl: 0.99-12.13, respectively). Appendix 2 and 3 shows the unadjusted associations for all background characteristics, the adjusted associations before accounting 
for specific side-effects, and models with each side-effect modeled separately. Associations are consistent in direction and generally consistent in significance.

\section{Discussion}

We found that about one-third of women experienced side-effects while using hormonal methods or the non-hormonal IUD, and that their associations with switching and discontinuation varied substantially based on the nature of the side-effect. Experiencing more bleeding was strongly associated with discontinuation, and irregular bleeding, physical discomfort, and vaginal dryness/lowered libido were associated with discontinuation. These results underscore the importance of examining the unique influence of specific side-effects, instead of broad singular category of "side-effects and health concerns", in studies of contraceptive practices. Failure to investigate specific contraceptive side-effects inhibits understanding of nuanced relationships between women's experiences and their adaptive responses in terms of contraceptive behaviors.

Our finding that increased menstrual bleeding was associated with discontinuation aligns with a growing body of literature, highlighting the importance of contraceptive-induced bleeding changes $(1,21)$. Excessive bleeding arose as a major barrier to contraceptive use in previous qualitative research in Uganda $(18,37,38)$; that excessive bleeding was strongly associated with discontinuation, but not with switching, indicates that women who experience this side-effect may be reluctant to switch to new methods, instead preferring to discontinue use altogether. The experience of excessive bleeding may present practical challenges to menstrual hygiene management. While limited, research in Uganda has highlighted a range of barriers to effective menstrual hygiene management, resulting from resource limitations and sociocultural norms (39-42). Despite the conceptual link between contraceptive induced bleeding changes and menstrual hygiene management, however, there is extremely limited research available on this subject, as the majority of studies related to menstrual health focus on the experience of adolescent girls (42). The experience of excessive bleeding may be additionally problematic in environments like Uganda, where myths and misperceptions around the harmful impacts of contraception, including effects on future fertility and on overall health, are widespread $(18,43,44)$. In high fertility countries such as Uganda, where childbearing is highly prized, excessive bleeding may not be viewed simply as an inconvenience, but as a prequel to infertility $(12,18)$. This belief carries profound social consequences and, therefore, may greatly reduce motivation to use contraception $(12,45)$.

We did not find significant effects with discontinuation or switching among women who reported less bleeding, although irregular bleeding was shown to reduce the risk of switching. Evidence from qualitative studies in Africa suggests that amenorrhea and irregular bleeding are often viewed negatively and associated with concerns about future fertility, particularly among nulliparous women (21), however recent qualitative evidence in Uganda found that the potential for lighter periods as a side-effect of 
contraception was viewed favorably by women (38). Of note, however, we did not distinguish between less bleeding and cessation of bleeding. Future research should attempt to distinguish between these two bleeding patterns, as complete cessation may engender different behavioral responses than a lighter, but consistent, menstrual period.

Though experiencing vaginal dryness/lowered libido and physical discomfort were only marginally statistically significant, both were associated with increased risk of switching and vaginal dryness/lowered libido demonstrated a particularly large effect size. Very little research has explored either quantitatively or qualitatively how changes to sexual experience induced by contraceptive use affect contraceptive dynamics, despite evidence that this is an important consideration for both the woman and her partner $(16,18,22,46)$. Similarly, physical discomfort, which here included headache, nausea, weakness, and cramping, have generally not been included in studies that assess the impact of side-effects. Unlike our findings, Barden-O'Fallon and colleagues found that abdominal cramping was not associated with the decision to switch versus stop method use. However, the authors did not assess whether cramping increased the overall risk of either relative to continuation (32). This limits our ability to compare our findings to other studies and underscores the critical need to explore these areas of contraceptive use and experience more fully.

A secondary objective of this paper was to gain a better understanding of the characteristics of women who continue, discontinue, and switch methods after experiencing side-effects, in order to explore how life circumstances influence contraceptive decision-making. Due to sample size limitations, we could not limit our analyses to only women who reported experiencing a side-effect, however, we were able to identify several relevant sociodemographic characteristics associated with discontinuation and switching, after adjusting for the experience of specific side-effects. Wealth was associated with decreased relative risk of discontinuation, while education was marginally associated with an increased risk of switching, indicating that social privilege may influence contraceptive dynamics in different ways. For example, social disadvantages that inhibit poorer women from seeking healthcare services to adopt new methods may result in higher discontinuation among this population. Educated women may be more knowledgeable about a range of contraceptive methods than less educated women, allowing them greater opportunity to switch methods if they are dissatisfied. More research on what influences women to discontinue versus switch contraceptive methods after experiencing side-effects is necessary so that family planning programs can better tailor services.

As noted in the methods, we did not restrict our sample based on fertility intention nor did we restrict the outcome to discontinuation or switching due to side-effects. Rather, our objective was to see if the experience of side-effects was still associated with contraceptive continuation, after adjusting for 
baseline fertility intentions. As the desire to avoid pregnancy may affect how willing women are to tolerate side-effects, we felt that this distinction was important to retain. Women who wanted to delay or limit childbearing by more than two years were significantly less likely to discontinue use than women who wanted a child within two years, but we observed little relationship with switching. This indicates that, while fertility intentions impact if a method is used at all, fertility intentions may have less influence on the specific method a woman chooses. Qualitative evidence from Uganda highlights the significant role that motivation to avoid pregnancy plays on contraceptive continuation, even when negative sideeffects are experienced, but there is little research that explores how fertility desires influence the decision to switch methods rather than discontinue (18).

A potential limitation of our study is that women were asked to spontaneously report side-effects that they were experiencing, rather than replying directly to a list of potential side-effects. To do so, women needed to consider what they were experiencing as a side-effect of their contraceptive method and, in the case of amenorrhea and loss of libido, recognize the absence or reduction of these experiences as being currently experienced. It is possible that women are less likely to report something that is not happening as being "currently experienced", relative to something that has increased or newly presented, but this requires further investigation. Reading a list of potential side-effects and requiring a response may be a more effective way to gather information on prevalence of side-effects, but may also result in overreporting $(47,48)$. This calls for further methodological research to identify how best to accurately identify and quantify the experience of side-effects in large-scale, population based surveys.

Under-reporting, in addition to our lower than expected retention, may have contributed to our limited analytical sample size. The limited absolute numbers of women, particularly for switching, restricted our ability to explore relationships across the full range of side-effects. Additionally, our retention at study level was $67 \%$. The application of propensity scores to adjust for loss-to-follow-up allowed us to construct weights that generated estimates of sample characteristics that did not differ between the full baseline and the sample of women who successfully completed follow-up. While we believe we thus adjusted for the majority of demographic and economic characteristics that may be related to contraceptive continuation, it is possible that there are unadjusted confounders that differ between these groups and may affect the overall generalizability of our findings. Finally, we defined switching and discontinuation only based on baseline and follow-up and were unable to account for events that occurred between the two time points, including both the onset of side-effects and switching or discontinuation in the interim. Though we included a contraceptive calendar for this purpose, we found that recall of starting and ending dates of methods when reported retrospectively was inconsistent and rendered the calendar data unusable for this purpose. As we were unable to account for the month-bymonth changes between the two surveys, we could have missed behaviors (additional discontinuation/switching of methods) that occurred between the two time points. We believe that this 
means our estimates are likely conservative, i.e. that we are under-estimating discontinuation and switching and over-estimating continuation.

Despite these limitations, our study has several important strengths. First, we were able to identify and assess the effect of a range of side-effects, including some which have not been extensively studied in the literature. Additionally, we used longitudinal data, following up with women one year after the baseline survey, enabling us to assess how these specific side-effects related to discontinuation and switching independently, providing additional insight into contraceptive behaviors.

\section{Conclusion}

Women do not experience generalized "side-effects and health concerns"; they experience specific sideeffects and contraceptive behavior is influenced based on both the side-effect and on the woman's unique circumstances and context. Identifying which side-effects are commonly experienced and strongly associated with discontinuation and switching, in addition to identifying some of the sociodemographic characteristics that may be associated with these behaviors, has valuable programmatic purposes. Counseling that incorporates discussions about individualized suitability for specific sideeffects and more discussion about the possibility of switching methods when side-effects do occur may improve continuation rates. Additional research should explore in greater detail the role that context, including partner support, plays in the decision to stop or switch methods.

\section{Declarations}

\section{Ethics approval and consent to participate}

All data collection procedures were approved by Institutional Review Boards (IRBs) at the Makerere University School of Public Health and Uganda National Council of Sciences and Technology in Kampala, Uganda, and the Johns Hopkins Bloomberg School of Public Health in Baltimore, USA. After eligibility was confirmed, women were asked to complete written consent; if the woman agreed to participate, she was then interviewed by a trained resident interviewer. For unmarried women aged 15-17, written consent was first obtained from a parent or legal guardian prior to the women providing written assent to participate.

\section{Consent for publication}

Not applicable 


\section{Availability of data and materials}

The datasets supporting the conclusions of this article are available at www.pmadata.org. All data are available upon request.

\section{Competing interests}

The authors declare that they have no competing interests

\section{Author's contributions}

LZ conceptualized the research question, led analysis, and was responsible for drafting the manuscript. DS assisted in all stages of conceptualization, analysis, and writing. CK and SW were major contributors to interpretation of results and writing. SA advised on all aspects of study design, analysis, and provided substantial edits to the manuscript. FM and SK led study implementation, provided interpretation of results, and substantive edits to the manuscript. All authors read and approved the final manuscript.

\section{Funding}

This research was funded by the Bill and Melinda Gates Foundation (grant OPP1163880). The funders had no role in the design of the study, collection, analysis, interpretation of data, orin writing the manuscript.

\section{Acknowledgements}

The authors thank Caroline Moreau for helpful comments and suggestions and Amy Tsui for supervision and oversite.

\section{References}

1. Castle S, Askew I. Contraceptive discontinuation:reasons, challenges, and solutions. Washington, DC: Population Council and FP2020; 2015 p. 41.

2. Bradley SEK, Schwandt HM, Khan S. Levels, trends, and reasons for contraceptive discontinuation [Internet]. Calverton, Maryland, USA: ICF Macro; 2009. Available from: http://dhsprogram.com/pubs/pdf/AS20/AS20.pdf 
3. Gezginc DK, Balci O, Karatayli R, Colakoglu MC. Contraceptive efficacy and side-effects of Implanon ${ }^{\circledR}$. The European Journal of Contraception \& Reproductive Health Care. 2007 Jan 1;12(4):362-5.

4. Lawrie TA, Helmerhorst FM, Maitra NK, Kulier R, Bloemenkamp K, Gülmezoglu AM. Types of progestogens in combined oral contraception: effectiveness and side-effects. Cochrane Database of Systematic Reviews [Internet]. 2011 [cited 2020 Mar 27];(5). Available from: https://www.cochranelibrary.com/cdsr/doi/10.1002/14651858.CD004861.pub2/abstract

5. Kaunitz AM. Long-acting injectable contraception with depot medroxyprogesterone acetate. American Journal of Obstetrics and Gynecology. 1994 May 1;170(5, Part 2):1543-9.

6. Reid RL, Fortier MP, Smith L, Mirkin S, Grubb GS, Constantine GD. Safety and bleeding profile of continuous levonorgestrel $90 \mathrm{mcg} /$ ethinyl estradiol $20 \mathrm{mcg}$ based on 2 years of clinical trial data in Canada. Contraception. 2010 Dec 1;82(6):497-502.

7. Harper CC, Rocca CH, Darney PD, von Hertzen H, Raine TR. Tolerability of levonorgestrel emergency contraception in adolescents. American Journal of Obstetrics and Gynecology. 2004 Oct 1;191(4):1158-63.

8. Sivin I, Mahgoub SE, McCarthy T, Mishell DR, Shoupe D, Alvarez F, et al. Long-term contraception with the levonorgestrel $20 \mathrm{mcg} /$ day ( $\mathrm{LNg} \mathrm{20}$ ) and the Copper T 380Ag intrauterine devices: A five-year randomized study. Contraception. 1990 Oct 1;42(4):361-78.

9. Sanders SA, Graham CA, Bass JL, Bancroft J. A prospective study of the effects of oral contraceptives on sexuality and well-being and their relationship to discontinuation. Contraception. 2001;64(1):51-8.

10. Tiihonen M, Leppänen H-M, Heikkinen A-M, Ahonen R. Hormonal Contraceptive Users' Self-Reported Benefits, Adverse Reactions, and Fears in 2001 and 2007. Patient-Patient-Centered-Outcome-Res. 2008 Jul 1;1(3):173-80.

11. Diamond-Smith N, Campbell M, Madan S. Misinformation and fear of side-effects of family planning. Culture, Health \& Sexuality. 2012 Apr;14(4):421-33.

12. Schwarz J, Dumbaugh M, Bapolisi W, Ndorere MS, Mwamini M-C, Bisimwa G, et al. "So that's why I'm scared of these methods": Locating contraceptive side-effects in embodied life circumstances in Burundi and eastern Democratic Republic of the Congo. Soc Sci Med. 2019;220:264-72.

13. Bawah AA, Akweongo P, Simmons R, Phillips JF. Women's Fears and Men's Anxieties: The Impact of Family Planning on Gender Relations in Northern Ghana. Studies in Family Planning. 1999;30(1):5466.

14. Campbell M, Sahin-Hodoglugil NN, Potts M. Barriers to fertility regulation: a review of the literature. Stud Fam Plann. 2006 Jun;37(2):87-98.

15. Hyttel M, Rasanathan JJK, Tellier M, Taremwa W. Use of injectable hormonal contraceptives: diverging perspectives of women and men, service providers and policymakers in Uganda. Reprod Health Matters. 2012 Dec;20(40):148-57. 
16. Jain A, Reichenbach L, Ehsan I, Rob U. "Side-effects affected my daily activities a lot": a qualitative exploration of the impact of contraceptive side-effects in Bangladesh. Open Access J Contracept. 2017;8:45-52.

17. Darroch JE, Sedgh G, Ball H. Contraceptive Technologies: Responding to Women's Needs. :52.

18. Kibira SPS, Muhumuza C, Bukenya JN, Atuyambe LM. "I Spent a Full Month Bleeding, I Thought I Was Going to Die..." A Qualitative Study of Experiences of Women Using Modern Contraception in Wakiso District, Uganda. PLoS One [Internet]. 2015 Nov 2 [cited 2020 May 14];10(11). Available from: https://www.ncbi.nlm.nih.gov/pmc/articles/PMC4629884/

19. Uganda Bureau of Statistics (UBOS), ICF. Uganda Demographic and Health Survey 2016. Kampala, Uganda and Rockville, Maryland, USA: UBOS and ICF; 2018.

20. Makerere University, School of Public Health at the College of Health Sciences, Bill and Melinda Gates Institute for Population and Reproductive Health at the Johns Hopkins Bloomberg School of Public Health. Performance Monitoring and Accountability 2020 (PMA2020) Survey Round 6, PMA2018/Uganda-R6. Uganda and Baltimore, Maryland, USA; 2018.

21. Polis CB, Hussain R, Berry A. There might be blood: a scoping review on women's responses to contraceptive-induced menstrual bleeding changes. Reproductive Health [Internet]. 2018 Dec [cited 2018 Sep 28];15(1). Available from: https://reproductive-health-

journal.biomedcentral.com/articles/10.1186/s12978-018-0561-0

22. Wood SN, Karp C, Zimmerman L. Women's sexual experiences as a side-effect of contraception in low- and middle-income countries: evidence from a systematic scoping review. Sex Reprod Health Matters. 2020 Dec;28(1):1763652.

23. Cristobal I, Lete LI, Viuda E de la, Perulero N, Arbat A, Canals I. One year quality of life measured with SEC-QoL in levonorgestrel 52 mg IUS users. Contraception. 2016 Apr;93(4):367-71.

24. Wong RC, Bell RJ, Thunuguntla K, McNamee K, Vollenhoven B. Implanon users are less likely to be satisfied with their contraception after 6 months than IUD users. Contraception. 2009 Nov 1;80(5):452-6.

25. Stephenson J, Shawe J, Panicker S, Brima N, Copas A, Sauer U, et al. Randomized trial of the effect of tailored versus standard use of the combined oral contraceptive pill on continuation rates at 1 year. Contraception. 2013 Oct 1;88(4):523-31.

26. Capurchande R, Coene G, Schockaert I, Macia M, Meulemans H. "It is challenging... oh, nobody likes it!": a qualitative study exploring Mozambican adolescents and young adults' experiences with contraception. BMC Womens Health [Internet]. 2016 Jul 30 [cited 2018 Sep 17];16. Available from: https://www.ncbi.nlm.nih.gov/pmc/articles/PMC4967333/

27. Abdel-Tawab N, RamaRao S. Do improvements in client-provider interaction increase contraceptive continuation? Unraveling the puzzle. Patient Educ Couns. 2010 Nov 12;81(3):381-7.

28. Ali MM, Cleland J. Contraceptive Switching after Method-related Discontinuation: Levels and Differentials. Studies in Family Planning. 2010 Jun 7;41(2):129-33. 
29. Ali MM, Cleland J, Shah IH. Causes and consequences of contraceptive discontinuation Evidence from 60 demographic and health surveys [Internet]. World Health Organization; 2012. Available from: https://www.who.int/reproductivehealth/publications/family_planning/9789241504058/en/

30. Steele F, Curtis SL, Choe M. The impact of family planning service provision on contraceptive-use dynamics in Morocco. Stud Fam Plann. 1999 Mar;30(1):28-42.

31. RamaRao S, Mohanam R. The quality of family planning programs: concepts, measurements, interventions, and effects. Stud Fam Plann. 2003 Dec;34(4):227-48.

32. Barden-O'Fallon J, Speizer I. What Differentiates Method Stoppers from Switchers? Contraceptive Discontinuation and Switching Among Honduran Women. International Perspectives on Sexual and Reproductive Health. 2011 Mar;37(01):016-23.

33. World Population Prospects - Population Division - United Nations [Internet]. [cited 2020 Oct 23]. Available from: https://population.un.org/wpp/

34. Korn EL, Graubard BI. Epidemiologic studies utilizing surveys: accounting for the sampling design. Am J Public Health. 1991 Sep;81(9):1166-73.

35. StataCorp. Stata Statistical Software: Release 16. College Station, TX: StataCorp LLC; 2019.

36. von Elm E, Altman D, Egger M, Pocock S, Gotzsche P, Vandenbroucke J. The Strengthening the Reporting of Observational Studies in Epidemiology (STROBE) Statement: guidelines for reporting observational studies.

37. Morse JE, Rowen TS, Steinauer J, Byamugisha J, Kakaire O. A qualitative assessment of Ugandan women's perceptions and knowledge of contraception. International Journal of Gynecology \& Obstetrics. 2014;124(1):30-3.

38. Callahan RL, Brunie A, Mackenzie ACL, Wayack-Pambè M, Guiella G, Kibira SPS, et al. Potential user interest in new long-acting contraceptives: Results from a mixed methods study in Burkina Faso and Uganda. PLOS ONE. 2019 May 28;14(5):e0217333.

39. Boosey R, Prestwich G, Deave T. Menstrual hygiene management amongst schoolgirls in the Rukungiri district of Uganda and the impact on their education: a cross-sectional study. The Pan African Medical Journal [Internet]. 2014 Jul 11 [cited 2020 Oct 22];19(253). Available from: https://www.panafrican-med-journal.com/content/article/19/253/full/

40. Hennegan J, Nansubuga A, Smith C, Redshaw M, Akullo A, Schwab KJ. Measuring menstrual hygiene experience: development and validation of the Menstrual Practice Needs Scale (MPNS-36) in Soroti, Uganda. BMJ Open. 2020 Feb 1;10(2):e034461.

41. Miiro G, Rutakumwa R, Nakiyingi-Miiro J, Nakuya K, Musoke S, Namakula J, et al. Menstrual health and school absenteeism among adolescent girls in Uganda (MENISCUS): a feasibility study. BMC Women's Health. 2018 Jan 3;18(1):4.

42. Hennegan J, Shannon AK, Rubli J, Schwab KJ, Melendez-Torres GJ. Women's and girls' experiences of menstruation in low- and middle-income countries: A systematic review and qualitative metasynthesis. PLoS Med. 2019;16(5):e1002803. 
43. Sedlander E, Bingenheimer JB, Thiongo M, Gichangi P, Rimal RN, Edberg M, et al. "They Destroy the Reproductive System": Exploring the Belief that Modern Contraceptive Use Causes Infertility. Studies in Family Planning. 2018;49(4):345-65.

44. Gueye A, Speizer IS, Corroon M, Okigbo CC. Belief in Family Planning Myths at the Individual And Community Levels and Modern Contraceptive Use in Urban Africa. Int Perspect Sex Reprod Health. 2015 Dec;41(4):191-9.

45. Dumbaugh M, Bapolisi W, Bisimwa G, Mwamini M-C, Mommers P, Merten S. Navigating fertility, reproduction and modern contraception in the fragile context of South Kivu, Democratic Republic of Congo: "Les enfants sont une richesse." Cult Health Sex. 2019;21(3):323-37.

46. Higgins JA, Smith NK. The Sexual Acceptability of Contraception: Reviewing the Literature and Building a New Concept. The Journal of Sex Research. 2016 May 3;53(4-5):417-56.

47. Kalton G, Schuman H. The Effect of the Question on Survey Responses: A Review. Journal of the Royal Statistical Society Series A (General). 1982;145(1):42-73.

48. Friborg $\mathrm{O}$, Rosenvinge $\mathrm{JH}$. A comparison of open-ended and closed questions in the prediction of mental health. Qual Quant. 2013 Apr 1;47(3):1397-411.

\section{Tables}

The tables are accessible in the Supplementary Files.

\section{Figures}




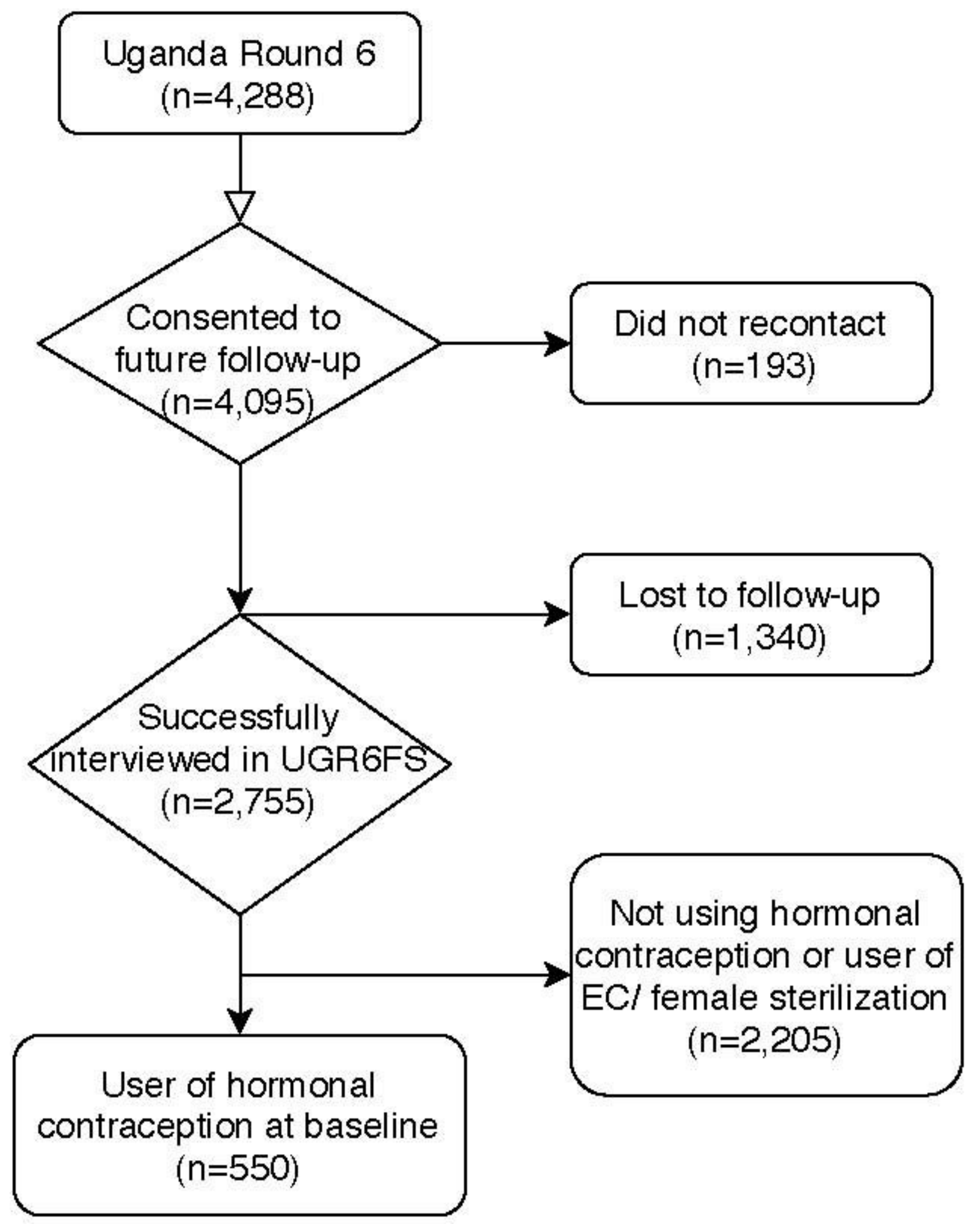

Figure 1

Selection Process for Analytic Sample

\section{Supplementary Files}

This is a list of supplementary files associated with this preprint. Click to download. 
- completedSTROBEcohortchecklist.docx

- Table1.docx

- Table2.docx

- Table3.docx

- Table4.docx 